\title{
A Split Mouth Randomized Clinical Comparative Study to Evaluate the Efficacy of Gingival Depigmentation Procedure Using Conventional Scalpel Technique or Diode Laser
}

\author{
Girish Suragimath", Mohini Hemant Lohana, Siddhartha Varma \\ Department of Periodontology, School of Dental Sciences, KIMSDU, Karad, Maharashtra, India
}

\author{
*Correspondence to \\ Girish Suragimath, MDS \\ Department of Periodontology, \\ School of Dental Sciences, KIMSDU, \\ Karad, Maharashtra State, India. Pin \\ code-415110; \\ Tel: +918007467216 ; \\ Email: \\ drgirishsuragimath@gmaill.com
}

Published online 27 October 2016

\begin{abstract}
Introduction: Dark or black coloured gingiva is an esthetic concern especially in subjects with high lip line or gummy smile. Gingival depigmentation procedure is a type of perioplastic surgery where the gingival epithelium is excised with various techniques to lighten the colour of the gingiva. The aim of this study was to compare the clinical efficacy of gingival depigmentation procedure with conventional scalpel technique and diode laser application. Methods: This split mouth randomized study was conducted on 12 subjects (18-40 years of age), exhibiting melanin hyperpigmentation of gingiva. The anterior labial sextant of maxilla and mandible were divided into two halves involving three anterior teeth i.e. central incisor, lateral incisor and canine on each side. The divided areas were randomly allotted for depigmentation procedure either with scalpel technique or diode laser operating at $980 \mathrm{~nm}$ wavelength. Various parameters such as bleeding, pain, difficulty of procedure and wound healing were assessed and compared between the two techniques. The level of melanin pigment was assessed with Dummette Gupta index and photographic analysis with the help of adobe software. The subjects were followed up to one year to see for recurrence of melanin pigmentation.

Results: Bleeding during surgery, pain score and difficulty of procedure assessed by the operator were statistically higher for scalpel technique as compared to laser technique. Wound healing did not show any statistical significant difference between both techniques. Gingival depigmentation procedures with scalpel as well as laser technique were effective when compared preoperatively and at consecutive postoperative visits, and this was statistically significant. Comparison of melanin depigmentation procedure between scalpel and laser technique did not show any significant differences at all postoperative intervals.

Conclusion: The findings of the present study suggest that gingival depigmentation was effective with both scalpel and laser techniques. However, the laser treated sites showed reduced pain experienced by the patient and better operator comfort. Slight melanin repigmentation was observed in three subjects treated with scalpel depigmentation procedure at the end of one year.

Keywords: Depigmentation; Diode laser; Pigmentation; Repigmentation; Gingiva.
\end{abstract}

\section{Introduction}

A smile is an expression denoting pleasure, sociability, happiness or amusement and can reflect self-confidence of an individual. A smile seems to have a favorable influence upon others and makes one likeable and more approachable. The beauty of a smile is not only influenced by how the teeth and lips look, but also by the way gingival tissue appears. A dark or black colored gum is one of the concerns of patients reporting to dental clinics. Dark pigmentation of the gum usually occurs due to excessive melanin deposition in gingival epithelium. Mel- anin pigmentation of the gingiva is a physiologic process that occurs in all ethnicities. ${ }^{1}$ The prevalence of melanin pigmentation varies between $0 \%$ to $89 \%$ in different populations with regard to ethnic factors and tobacco usage. ${ }^{2}$ Gingival hyperpigmentation is termed as physiological or racial pigmentation, as it occurs as a genetic trait in some populations. ${ }^{3}$ Gingival hyperpigmentation in patients with gummy smile or excessive gingival display could pose an esthetic problem. Gingival depigmentation is a periodontal plastic procedure carried out by various techniques like conventional scalpel technique, gingivec-

Please cite this article as follows: Suragimath G, Lohana $\mathrm{MH}$, Varma S. A split mouth randomized clinical comparative study to evaluate the efficacy of gingival depigmentation procedure using conventional scalpel technique or diode laser. J Lasers Med Sci. 2016;7(4):227232. doi:10.15171/jlms.2016.40. 
tomy, ${ }^{4}$ free gingival graft, bur abrasion, surgical scraping, cryotherapy, ${ }^{5}$ electrosurgery ${ }^{6}$ and laser therapy. ${ }^{7}$ The choice of surgical technique for depigmentation is based on individual preferences and clinician's experience.

Lasers are being widely used in dentistry for various treatment modalities since the beginning of the 1980s. Semiconductor diode lasers have been used for frenectomy, gingivectomy, biopsy, operculectomy and implant dentistry. Lasers have been reported as reliable, safe and effective with minimal postoperative discomfort. ${ }^{8}$ The present study was carried out to assess and compare the efficacy of gingival depigmentation procedure using conventional scalpel technique and diode laser.

\section{Methods}

This split mouth randomized comparative clinical trial was carried out between September 2014 and June 2015 in the Department of Periodontology, School of Dental Sciences (SDS), Krishna Institute of Medical Sciences (KIMSDU), Karad.

\section{Patient Selection}

Twelve subjects in the age range of 18 to 40 years (21.66 \pm 8.51$)$ complaining of unaesthetic dark gums were selected for the study. The sample size for the study was determined by power analysis. Gingival depigmentation procedure was planned in anterior labial gingiva of maxillary and mandibular quadrant.

This split mouth study was carried out to achieve depigmentation in the anterior esthetic zone i.e. incisors to canine (Figure 1). The subjects were informed about the objectives of the study and an informed consent was

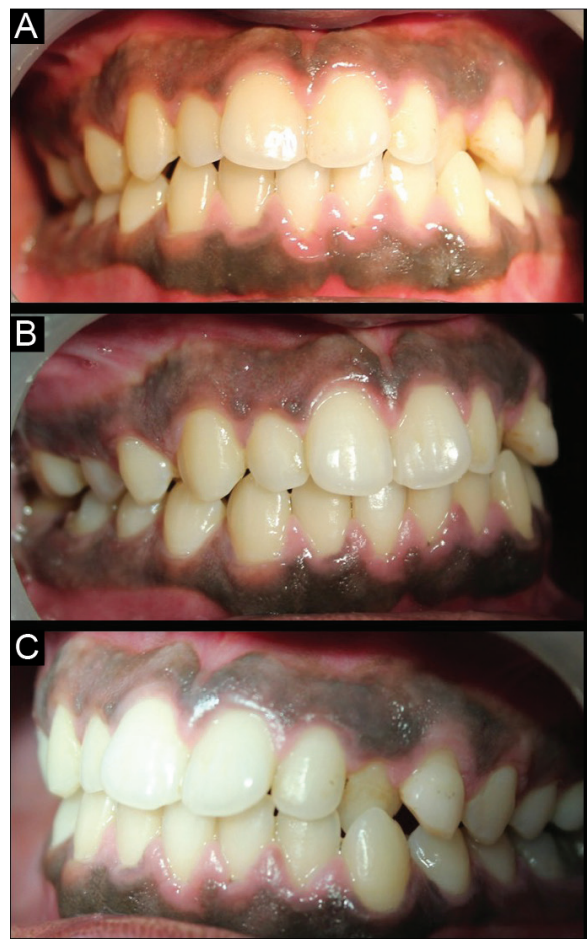

Figure 1. Preoperative Photograph: (A) Frontal, (B) Right Lateral, and (C) Left Lateral Views. obtained before enrolling them. Pigmentation in anterior sextant labial side of maxillary and mandibular arch from canine to canine was divided into two halves involving three anterior teeth i.e. central incisor, lateral incisor and canine on each side. The divided areas were randomly allotted for depigmentation procedure either with scalpel technique or diode laser by flip of a coin.

Dummett-Gupta oral pigmentation (DOP) index ${ }^{9}$ was used to grade the level of gingival hyper pigmentation. Scoring criteria for DOP

1: No clinical pigmentation.

2: Mild clinical pigmentation.

3: Moderate clinical pigmentation.

4: Heavy clinical pigmentation.

Exclusion criteria: Pregnant and lactating women, medically compromised subjects, smokers and subjects having history of postsurgical keloid. Patients in whom, gingival hyperpigmentation was associated with other syndromes, lesions and conditions.

All enrolled patients underwent oral prophylaxis and were asked to follow oral hygiene instructions. The depigmentation procedure was performed using conventional scalpel or diode laser, and an interval of one week was kept between the two procedures to assess the pain and healing pattern. The patients were followed up to one year to assess for any sign of recurrence of melanin pigmentation.

\section{Conventional Scalpel Technique}

The area was anesthetized with $2 \%$ lignocaine hydrochloride (LOX 2\%, Neon laboratories Ltd, Andheri Mumbai). Partial thickness flap was excised with a surgical blade no. 15 and complete gingival epithelium was removed. The depigmentation was carried out from the mucogingival junction to the marginal gingiva (Figure 2). Additional measures were taken to ensure that all the pigmented epithelium was removed. Hemorrhage during the surgical procedure was controlled by direct pressure with sterile gauze. The depigmented area was covered with a periodontal dressing (COE -PAK, GC America Inc., ALSIP, IL, USA).

\section{Depigmentation With Diode Laser}

The area was anesthetized with $2 \%$ lignocaine hydrochloride (LOX 2\%, Neon laboratories Ltd, Andheri Mumbai). The depigmentation was carried out with diode laser with $980 \mathrm{~nm}$ wavelength (Photon Plus; Zolar Tech Technology Co Inc., Canada). The laser beam was operated with

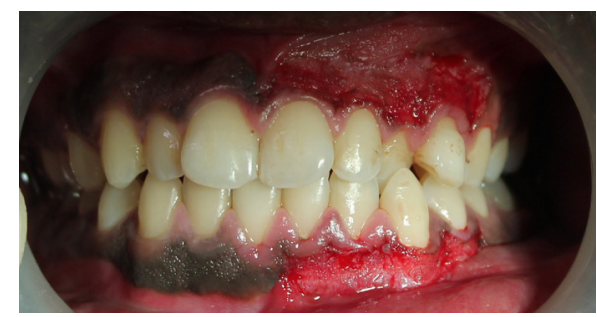

Figure 2. Depigmentation Procedure Using Scalpel Technique on the Left Quadrant. 
the following parameters: continuous wave mode, initiated tip, power setting of $0.5 \mathrm{~W}$ and energy $120 \mathrm{~mJ}$ (Table 1). Laser safety protocols were followed as per Food and Drug Administration (FDA) guidelines. The laser tip was used in contact mode on the pigmented part of gingiva. The ablation was performed using paint brush strokes from the mucogingival junction to the free gingival margin (Figure 3). Sterile gauze soaked in saline was used to remove the char formed over the surface of the surgical area. Thorough examination was carried out to make sure that all pigmented epithelium were removed. The depigmented area was covered with a periodontal dressing (COE -PAK, GC America Inc, ALSIP, IL, USA).

Analgesics were prescribed to control pain and patients were asked to follow postoperative instructions. Patients were instructed to avoid eating of hot and spicy foods for the first 24 hours and were advised to use chlorhexidine mouthwash twice daily.

A single calibrated operator recorded clinical parameters like bleeding, pain, difficulty of procedure and wound healing as per the criteria of Ishii et $\mathrm{al}^{10}$ (Table 2).

\section{Method of Scoring}

The pain experienced during the procedure was recorded immediately after the procedure and also on the seventh

Table 1. Laser Parameters and Their Specification

\begin{tabular}{ll}
\hline Type of Laser & Diode $980 \mathrm{~nm}(10 \mathrm{~W})$ \\
\hline Emission mode & Continuous or pulsed \\
Time on/time off & $0.01 \mathrm{~ms}$ to $9.9 \mathrm{~s}$ \\
Delivery system & Fiberoptic \\
Power range & $100 \mathrm{~mW}$ to $10 \mathrm{~W}$ \\
Peak power & $10 \mathrm{~W}$ \\
Pulse duration & $0.1 \mathrm{~ms}$ to $9.9 \mathrm{~s}$ \\
Pulse frequency & Regulated from $1 \mathrm{~Hz}$ to $5000 \mathrm{~Hz}$ \\
\hline Duty cycle & Adjustable \\
Aiming beam & $650 \mathrm{~nm} 2 \mathrm{~mW}$ \\
Audible notification & Yes \\
Visual notification & Yes \\
Power requirement & AC100-240 V $50 / 60 \mathrm{~Hz}$ \\
\hline Battery & $\begin{array}{l}\text { Rechargeable lithium polymer } 3.7 \\
\text { (Photon) }\end{array}$ \\
\hline Wireless foot pedal & Frequency $2.4 \mathrm{GHz}$ \\
\hline
\end{tabular}

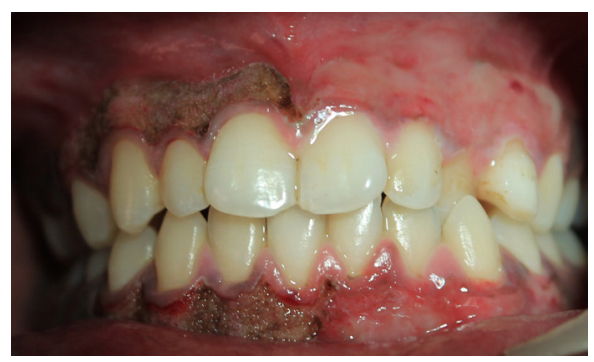

Figure 3. Depigmentation Procedure Using Diode Laser Technique on the Right Quadrant. day. The subjects were asked to rate the degree of pain, on a $10 \mathrm{~cm}$ horizontal visual analog scale (VAS) ${ }^{11}$ by placing a vertical mark to assess position between the two endpoints. Related to the pain scale, the left end point was nominated as "no pain" whereas the right end point was nominated as "unbearable pain," where VAS score: $0=$ no pain; 1-3 = slight pain; 3.1-6 = moderate pain; and 6.1-10 $=$ severe pain.

Bleeding during the procedure and postoperatively was assessed. Difficulty of procedure experienced by the operator was evaluated. Wound healing was assessed on day seven (Figure 4). Recurrence of melanin pigmentation was evaluated after 3, 6 and 12 months interval using DOP index.

Clinical photographs were captured using the SLR camera (Canon EOS 1100 D) with standardization (Resolution-12 megapixels, Distance-10 cm from the object and fixed magnification) for all patients on recall visits. After taking all pre and postoperative photographs, an examiner expert in using Photoshop software traced and measured the pigmented areas of all images with Photoshop software (CS3) (Adobe system, United States). Equal sized square areas in each segment of an image were selected and calculated in regard to the mean of red, green, blue (RGB) values (Figures 5). The mean RGB values of selected points were compared pre and postoperatively, at 3, 6 and 12 months intervals (Figure 6).

\section{Statistical Analysis}

Statistical analysis was carried out using SPSS version 17 for Windows (SPSS Inc., Chicago, USA). Non-parametric tests were used for continuous variables because the data were not distributed normally. Comparisons between the groups were performed using the Mann-Whitney U test. Results were represented as mean \pm standard deviation (SD) and $P$ value $<0.05$ was considered significant.
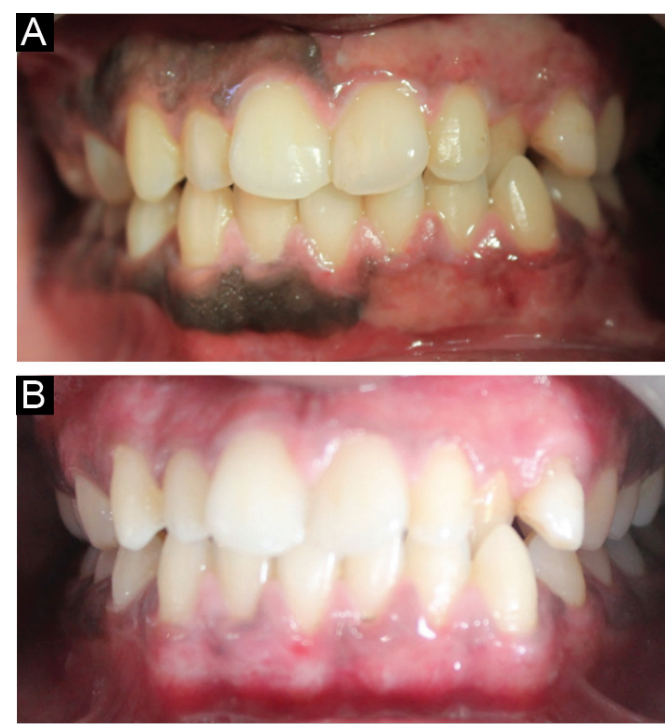

Figure 4. Assessing Wound Healing After One Week. (A) Scalpel treated area on the left quadrant (B) Laser treated area on the right quadrant. 
Table 2. The Criteria of Ishii et $\mathrm{al}^{10}$

\begin{tabular}{|c|c|c|c|c|}
\hline \multirow{2}{*}{ Evaluation } & \multicolumn{4}{|c|}{ Score } \\
\hline & 1 & 2 & 3 & 4 \\
\hline Bleeding & None & Slight & Moderate & Severe \\
\hline Pain & None & Slight & Moderate & Severe \\
\hline Difficulty of procedure & Very easy & Easy & Difficult & Impossible \\
\hline Wound healing & Complete epithelialization & Incomplete epithelialization & Ulcer & Tissue defect or necrosis \\
\hline
\end{tabular}

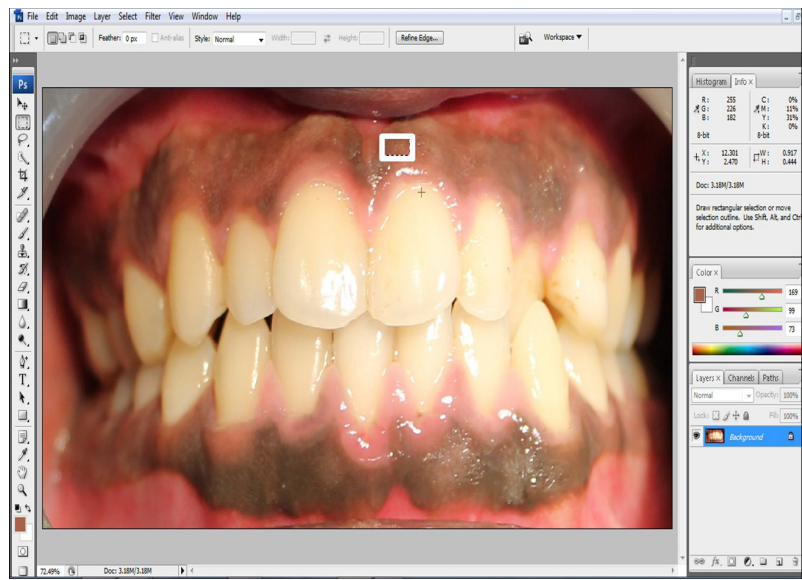

Figure 5. Photoshop Analysis of Selected Points.
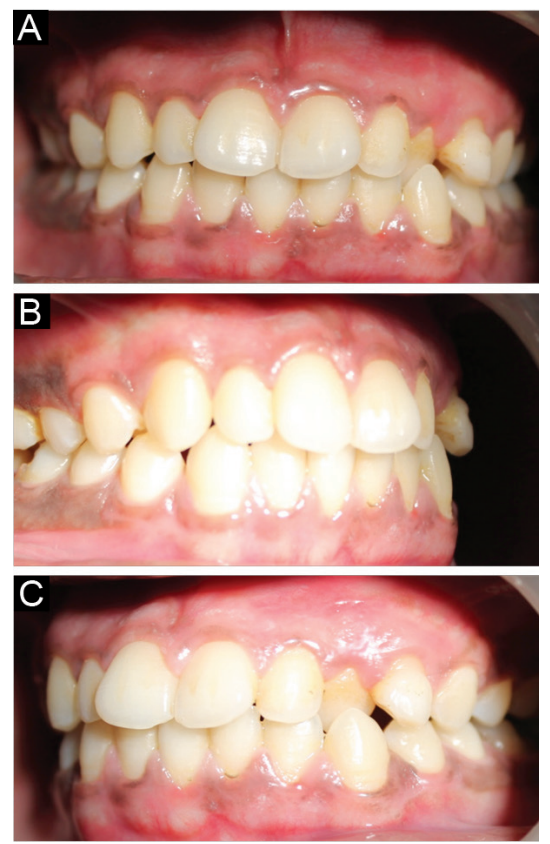

Figure 6. Postoperative Photograph at the End of One Year: (A) Frontal view, (B) Right lateral, (C) Left lateral view.

\section{Results}

Out of 12 subjects, 5 were females and 7 were males with age ranging from 18 to 40 years (mean age 21.66 \pm 8.5 ). Bleeding, pain, difficulty of procedure and wound healing were assessed as per the criteria Ishii et al. ${ }^{10}$

Bleeding during surgery was compared between both techniques. Laser treated areas showed relatively less bleeding compare to conventional scalpel, and the difference was statistically significant (Table 3 ).

Comparison of patients' pain experience as per VAS on day one and on the seventh day is presented in Table 4. Analysis showed that, VAS score of pain for laser procedure during surgery was significantly lower compared to the conventional scalpel procedure $(P<0.05)$, but on the seventh day, there was no difference observed between both techniques.

Procedure's difficulty was compared between both techniques. Laser depigmentation technique was easier to perform than conventional scalpel technique as experienced by the operator, and the difference was statistically significant (Table 5).

Comparison of wound healing at day one and after 7 days did not show any statistical significant difference between both techniques (Table 6).

Comparison of preoperative and postoperative melanin pigment with DOP index at one week, 3 months, 6 months and 12 months follow-up showed that there were statistically significant differences observed. The comparison of melanin pigment scores between scalpel and laser techniques showed that there was no statistical significant difference observed at all postoperative intervals between both techniques (Table 7).

Comparison of preoperative and postoperative photographic analysis using RGB values at one week, 3 months, 6 months and 12 months follow-up, showed that there was a statistically significant difference observed. Comparison of RGB values between scalpel and laser techniques showed that there was no statistical significant difference observed at all the postoperative intervals between both techniques. (Table 8).

Table 3. Bleeding During the Surgery Assessed by Operator

\begin{tabular}{llll}
\hline & Group & Mean \pm SD & P Value \\
\hline Bleeding during & Scalpel & $2.917 \pm 0.2887$ & $<0.001^{\text {a }}$ \\
surgery & Laser & $1.417 \pm 0.5149$ & $<0.001^{\text {a }}$ \\
\hline
\end{tabular}

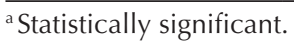

Table 4. Pain Perception for Scalpel and Laser Techniques

\begin{tabular}{llll}
\hline & Group & Mean \pm SD & P Value \\
\hline VAS score on & Scalpel & $3.5 \pm 0.7977$ & $0.001^{\text {a }}$ \\
day 1 & Laser & $1.5 \pm 0.5222$ & \\
VAS at Day 7 & Scalpel & $0.4167 \pm 0.5149$ & 0.1587 \\
& Laser & $0.0833 \pm 0.287$ & \\
\hline
\end{tabular}

a Statistically significant. 
Table 5. Difficulty of Procedure Assessed by the Operator

\begin{tabular}{llll}
\hline & Group & Mean \pm SD & P Value \\
\hline Difficulty of & Scalpel & $2.917 \pm 0.2887$ & $<0.001^{\mathrm{a}}$ \\
procedure & Laser & $1.2887 \pm 0.4523$ & $<0.001^{\mathrm{a}}$ \\
\hline a & &
\end{tabular}

a tatistically significant.

Table 6. Wound Healing Between Scalpel and Laser techniques

\begin{tabular}{llll}
\hline & Group & Mean \pm SD & P Value \\
\hline $\begin{array}{llll}\text { Wound healing } \\
\text { day } 1\end{array}$ & Scalpel & $1.083 \pm 0.2887$ & \\
\cline { 2 - 3 } Wound healing & Laser & $1.167 \pm 0.3892$ & 0.738 \\
day 7 & Scalpel & $1.088 \pm 0.2890$ & \\
& Laser & $1.174 \pm 0.3898$ & 0.835 \\
\hline
\end{tabular}

\section{Discussion}

Melanin hyperpigmented gingival tissue results in unaesthetic appearance of gums. It often forces patients to seek esthetic treatment to lighten the colour of gingiva. The use of several depigmentation modalities like simple slicing method to free gingival grafts or "push back" operation have been suggested in the literature. ${ }^{12}$

The absorption of laser light at 800 to $980 \mathrm{~nm}$ is poor in water, but high in hemoglobin and other pigments. ${ }^{13}$ The diode laser is an excellent soft tissue surgical laser as it does not interact with dental hard tissues. Thermal effects of diode laser are attributed to 'hot tip' effect caused by accumulation of heat at the end of fiber. These effects result in the production of a thick coagulation layer on the treated surface. The advantage of diode laser includes smaller and handy size of the unit, and also no detrimental effects on alveolar bone and root surface. The healing of diode laser wounds is slower and needs longer time than conventional scalpel wounds. However scalpel surgery results in unpleasant bleeding during and after operation and requires periodontal dressing to cover the surgical wound. A sterile inflammatory reaction has been observed in the gingival tissues following use of diode laser. ${ }^{14}$ Blood vessels in vicinity of diode laser tip are sealed, thus resulting in hemostasis and relatively clean operating field.

In our study, bleeding during surgery was assessed between both techniques. Laser treated areas showed relatively less bleeding than scalpel treated areas, and the difference was statistically significant. The findings of the present study are consistent with the studies conducted by Lagdive et $\mathrm{al}^{7}$ and Lee et al. ${ }^{15}$ This may be because laser has the ability to cut and coagulate tissues. The protein coagulum formed on the wound surface acts as a biological dressing and seals the ends of the capillaries and venules, reducing the bleeding during laser surgery.

Laser depigmentation procedure as compared to scalpel procedure had significantly less postoperative pain on day one. The increased pain perception associated with the scalpel might be attributed to the fact that it is a more intrusive surgical procedure involving blood loss and a wide open surgical wound. The open wound also contributes to the discomfort postoperatively since it heals by secondary intention. The findings of the present study are similar to the ones of the studies conducted by Ribeiro et $\mathrm{al}^{16}$ and Lagdive et $\mathrm{al},{ }^{7}$ who showed that the sites treated with laser had slight or no pain. On day seven, there was no statistical significant difference between both groups in terms of pain perception. The findings of the present study are similar to the study conducted by Schroeder, ${ }^{17}$ who concluded that postoperative pain experienced by the patients with laser treatment was less compared to scalpel technique.

Comparison of difficulty of procedure assessed by the operator showed that laser technique was easy to perform due to reduced bleeding during the operative surgery and was less technique sensitive compared to scalpel technique. The difference was highly statistically significant. The result of the current study is similar to the study conducted by Simşek Kaya et al. ${ }^{18}$

Wound healing was assessed on day one and after 7 days, scalpel treated areas healed faster compared to laser treated areas, but this did not reach a statistically significant level. The finding of the present study is consistent with the study conducted by Kasagani et al. ${ }^{19}$

Melanin pigmentation score was evaluated by DOP index preoperatively, 1 week, 3 months, 6 months and 12 months postoperatively. There was no statistical significant difference in the melanin scores between both techniques. There was a slight recurrence of repigmentation observed in three patients of scalpel group at the end of one year. The findings of the present study are in accordance with the study conducted by Perlmutter and Tal. ${ }^{20}$ The clinical reappearance of melanin pigment following a period of clinical depigmentation is referred as "Repigmentation." Postsurgical repigmentation of gingiva has been reported in the literature. ${ }^{21}$ Repigmentation is a spontaneous pro-

Table 7. Melanin Pigment Evaluation With Dummett-Gupta Index

\begin{tabular}{|c|c|c|c|c|c|}
\hline Procedure & Preoperatively & 7 Days & 3 Months & 6 Months & 12 Months \\
\hline Scalpel & $2.58 \pm 0.51$ & $0.16 \pm 0.38$ & $0.11 \pm 0.31$ & $0.23 \pm 0.36$ & $0.33 \pm 0.49$ \\
\hline Laser & $2.58 \pm 0.51$ & $0.08 \pm 0.28$ & $0.22 \pm 0.41$ & $0.31 \pm 0.48$ & $0.83 \pm 0.28$ \\
\hline$P$ value & 0.9764 & 0.7373 & 0.2510 & 0.2615 & 0.2912 \\
\hline
\end{tabular}

Table 8. Comparison of RGB Value Between Scalpel and Laser Technique

\begin{tabular}{lccccc}
\hline Procedure & Preoperatively & 7 Days & 3 Months & 6 Months & 12 Months \\
\hline Scalpel & $236.25 \pm 61.06$ & $411.33 \pm 53.25$ & $429.33 \pm 69.19$ & $424 \pm 104.89$ & $435.91 \pm 110.88$ \\
Laser & $205.83 \pm 90.95$ & $358.58 \pm 116.32$ & $363.33 \pm 79.16$ & $392.58 \pm 72.93$ & $417.41 \pm 86.79$ \\
$P$ value & 0.34 & 0.16 & 0.04 & 0.40 & 0.65 \\
\hline
\end{tabular}


cess and has been attributed to the activity and migration of melanocytic cells from surrounding areas. ${ }^{1}$

The digital photograph analysis for RGB values before and after depigmentation procedure did not show any significant difference in both techniques. The findings of the present study are similar to those of the study conducted by Farahmand et al. ${ }^{22}$

Within the limitation of our study, we can conclude that diode laser depigmentation procedure is safe and effective, with a better patient and operator comfort. There is a need for further longitudinal studies with larger sample size to establish the exact efficacy of laser technique over the conventional scalpel technique for depigmentation procedure.

\section{Future Perspective}

Further studies with larger sample size should be carried out to substantiate the results. Different lasing medium e.g. Neodymium-Doped Yttrium Aluminium Garnet (ND:YAG) laser, carbon dioxide $\left(\mathrm{CO}_{2}\right)$ laser can be used to compare and evaluate for depigmentation procedure.

\section{Conclusion}

The findings of the present study suggest that gingival depigmentation was effective with both scalpel and laser techniques. However, the laser treated sites showed reduced pain experienced by the patient. The operator comfort was better in case of laser technique compared to scalpel technique. Recurrence rate of gingival pigmentation was faster in scalpel technique as compared to laser technique.

\section{Ethical Consideration}

The research protocol was initially submitted to the institutional ethical committee and ethical clearance was obtained before commencing the study (Ref no. KIMSDU/ IEC/2014, dated 06/12/2014). The study was conducted in accordance with Consort 2010 checklist.

\section{Conflict of Interests}

Authors declare that they have no competing interests.

\section{References}

1. Dummett CO. Clinical observations on pigment variations in healthy oral tissues of the Negro. J Dent Res. 1945;24(1):713.

2. Dummett CO, Barens G. Oromucosal pigmentation: an updated literary review. J Periodontol. 1971;42:726-736.

3. Prinz H. Pigmentation of the oral mucous membrane. Dental Cosmos. 1932;72:554-561.

4. Tamizi M, Taheri M. Treatment of severe physiologic gingival pigmentation with free gingival autograft. Quintessence Int. 1996;27:555-8

5. Tal H, Landsberg J, Kozlovsky A. Cryosurgical depigmentation of the gingiva: a cryosurgical depigmentation of the gingiva. J Clin Periodontol. 1987;14:614-617.

6. Deepak P, Sunil S, Mishra R, Sheshadri. Treatment of gingival pigmentation: A case series. Indian J Dent Res. 2005;16:171-176. doi:10.4103/0970-9290.29901.

7. Lagdive S, Doshi Y, Marawar PP. Management of gingival hyperpigmentation using surgical blade and diode laser therapy: a comparative Study. J Oral Laser Appl. 2009;9;4147.

8. Moritz A, Gutknecht N, Doertbudak O, et al. Bacterial reduction in periodontal pockets through irradiation with diode laser: a pilot study. J Clin Laser Med Surg. 1997;15:3337.

9. Dummett CO. Physiologic pigmentation of the oral and cutaneous tissues in the negro. J Dent Res. 1946;25:421432. doi:10.1177/00220345460250060201.

10. Ishii S, Aoki A, Kawashima Y, Watanabe H, Ishikawa I. Application of an Er:YAG laser to remove gingival melanin hyperpigmentation: Treatment procedure and clinical evaluation. J Jpn Soc Laser Dent. 2002;13:89-96.

11. Steigmann S. The relationship between physiologic pigmentation of the skin and oral mucosa in Yemenite jews. Oral Surg Oral Med Oral Pathol. 1965;19(1):32-38. doi:10.1016/0030-4220(65)90212-4.

12. Cobb CM. Lasers in periodontics: a review of the literature. $J$ Periodontol. 2006;77(4):545-564. 10.1902/jop.2006.050417.

13. The Academy of Laser Dentistry Featured wavelength: diode - the diode laser in dentistry (Academy report). Wavelengths. 2000;8:13.

14. Ozbayrak S, Dumlu A, Ercalik-Yalcinkaya S. Treatment of melanin-pigmented gingiva and oral mucosa by $\mathrm{CO}_{2}$ laser. Oral Surg Oral Med Oral Pathol Oral Radiol Endod. 2000;90(1):14-15. doi:10.1067/moe.2000.106396.

15. Lee KM, Lee DY, Shin SI, Kwon YH, Chung JH, Herr Y. A comparison of different gingival depigmentation techniques: ablation by erbium: yttrium-aluminiumgarnet laser and abrasion by rotary instruments. $J$ Periodont Implant Sci. 2011;41(4):201-207. doi:10.5051/ jpis.2011.41.4.201.

16. Ribeiro FV, Cavaller CP, Casarin RC, et al. Esthetic treatment of gingival hyperpigmentation with Nd:YAG laser or scalpel technique: a 6 month RCT of patient and professional assessment. Lasers Med Sci. 2014;29:537-544. doi:10.1007/s10103-012-1254-5.

17. Schroeder HE. Melanin containing organelles in cells of the human gingival I. Epithelial melanocytes. J Periodont Res. 1969;4(1):1-18. doi:10.1111/j.1600-0765.1969.tb01940.x.

18. Simşek Kaya G, Yapici Yavuz G, Sümbüllü MA, Dayi E. A comparison of diode laser and Er: YAG lasers in the treatment of gingival melanin pigmentation. Oral Surg Oral Med Oral Pathol Oral Radiol. 2012;113(3):293-299. doi:10.1016/j.tripleo.2011.03.005.

19. Kasagani SK, Nutalapati R, Mutthineni RB. Esthetic depigmentation of anterior gingiva-A case series. N Y State Dent J. 2012;78:26-31.

20. Perlmutter $\mathrm{S}, \mathrm{Tal} \mathrm{H}$. Repigmentation of the gingiva following surgical injury. J Periodontol. 1986;57(1):48-50. doi:10.1902/jop.1986.57.1.48.

21. Mokeem SA. Management of gingival hyperpigmentation by surgical abrasion: report of three cases. Saudi Dent J. 2006;18:162-6.

22. Farahmand A, Abed AM, Mansouri Y. Clinical application of Er: YAG laser and cryosurgery in gingival depigmentation. World J Dent. 2014;5:102-108. 